\title{
Comment on Limitations on the superposition principle: superselection rules in non-relativistic quantum mechanics
}

\author{
Namit Anand ${ }^{1}$ \\ ${ }^{1}$ National Institute of Science Education and Research, Bhubaneswar, India \\ E-mail: namit.anand@niser.ac.in
}

\begin{abstract}
This is a comment to the paper, Limitations on the superposition principle: superselection rules in non-relativistic quantum mechanics by $\mathrm{C}$ Cisneros et al 1998 Eur. J. Phys. 19 237. doi:10.1088/0143-0807/19/3/005.

The proof that the authors construct for the limitation on the superposition of state vectors corresponding to different sectors of the Hilbert space, partitioned by a superoperator has a flaw as outlined below.
\end{abstract}

\section{Introduction}

In Ref. [1], section 2.4(Impossibility of superposing states belonging to different coherent sectors), the authors construct a proof using a superoperator $G$ that commutes with all observables of the system. The eigenstates of operator $\mathrm{G}$ are of the form $\left|g_{m} ; \alpha_{m}\right\rangle$ where $g_{m}$ and $\alpha_{m}$ are labels to distinguish different eigenvectors all of which are non-degenerate, i.e. $\left\langle g_{m} ; \alpha_{m} \mid g_{n} ; \alpha_{n}\right\rangle=0$ if $m \neq m$.

In the next section(2.4), they construct a vector $|u\rangle=\sum_{m} u_{m}\left|g_{m} ; \alpha_{m}\right\rangle$ superposing the eigenstates of $\mathrm{G}$ and claim that since $\mathrm{G}$ commutes with every other observable, $|u\rangle$ should be an eigenstate of every operator in a complete set of commuting observables of the system.

Now we know that if $G|a\rangle=a|a\rangle$ and $G|b\rangle=b|b\rangle$ are eigenvectors of an operator G, then $\lambda_{1}|a\rangle+\lambda_{2}|b\rangle$ is not an eigenvector unless they have the same eigenvalue since:

$$
G\left(\lambda_{1}|a\rangle+\lambda_{2}|b\rangle\right)=a \lambda_{1}|a\rangle+b \lambda_{2}|b\rangle \neq \lambda\left(\lambda_{1}|a\rangle+\lambda_{2}|b\rangle\right)
$$

(for some $\lambda$ ) unless $a=b$, which is not true in general and definitely not true for the hermitian operators with non-degenerate eigenvalues in the paper.

It is true that if $\mathrm{G}$ commutes with every other observable and has non-degenerate eigenvalues, then all such obervables share the same eigenvectors. But $|u\rangle$ itself is not an eigenvector of $\mathrm{G}$ as proved above. And so $|u\rangle$ cannot be an eigenvector of other observables too. To summarize, the proof of superselection rules proposed is thus is not valid for the case discussed by the authors. Whether a similar proof can be constructed is still open.

\section{References}

[1] C Cisneros et al 1998 Eur. J. Phys 19 237, doi:10.1088/0143-0807/19/3/005, Limitations on the superposition principle: superselection rules in non-relativistic quantum mechanics. 